\title{
Surgical repair of bilateral levator ani muscles with ultrasound guidance: comment
}

\author{
Ka Lai Shek • Hans Peter Dietz
}

Published online: 7 November 2012

(C) The International Urogynecological Association 2012

We read the paper titled "Surgical Repair of Bilateral Levator Ani Muscles with Ultrasound Guidance" by Rostaminia et al. [1] with great interest. I would like to draw the attention of the authors to two previous publications in the literature on levator repair, as we believe they would be of interest to the readers and are relevant to this case report [2, $3]$. In the former study published in 2007, the authors reported a case of repair failure in a woman with a large vaginal tear and levator avulsion diagnosed at the delivery suite [2]. An abstract was published last year on the outcomes of concomitant levator repair in 15 patients with levator avulsion having prolapse repair [3]. The approach and techniques were different from the index case report in that the repair was done with no ultrasound guidance, a piece of mesh was used to reinforce the muscle, and the repair was done via a low lateral colpotomy at the level of the hymenal remnant.

We consider levator avulsion easy to identify macroscopically, with no need for imaging guidance. However, we found the effect of levator repair on prolapse recurrence and hiatal area on Valsalva, a parameter significantly asso-

An author's reply to this comment is available at doi $10.1007 / \mathrm{s} 00192$ 012-1986-6.

\author{
K. L. Shek $\cdot$ H. P. Dietz \\ University of Sydney, \\ Sydney, NSW, Australia \\ K. L. Shek $(\triangle)$ \\ Nepean Clinical School, Nepean Hospital, \\ Penrith, NSW 2750, Australia \\ e-mail: shekkalai@yahoo.com.hk
}

ciated with both signs and symptoms of pelvic organ prolapse (POP), rather disappointing. It is plausible that in women with levator avulsion there often is microscopic trauma and functional muscle impairment, and therefore, levator repair may not be an effective measure to reduce prolapse recurrence.

We are not sure of the indication for performing levator repair in the index case report, and the authors have rightly pointed out that it remains to be proven whether preemptive repair will reduce future occurrence of POP. For the time being, it may be prudent to limit such experimental surgery to patients who present with a condition that is strongly associated with avulsion, i.e., prolapse of the anterior and central compartments.

Conflicts of interest H.P. Dietz was a consultant for American Medical Systems, Continence Control Systems, and Materna. He has accepted speaker fees from Astellas, General Electric, and American Medical Systems and has benefited from an educational grant by General Electric. K.L. Shek has no conflict of interest to declare

\section{References}

1. Rostaminia SSG, Quiroz LH (2012) Surgical repair of bilateral levator ani muscles with ultrasound guidance. Int Urogynecol J. doi:10.1007/s00192-012-1912-y

2. Dietz HP, Gillespie A, Phadke P (2007) Avulsion of the pubovisceral muscle associated with large vaginal tear after normal vaginal delivery at term. Aust NZ J Obstet Gynaecol 47:341-344

3. Dietz HP, Shek KL, Korda A (2011) Can levator avulsion be corrected surgically? Neurourol Urodyn 30:878-879 